\title{
Spectral asymmetry of phonon sideband luminescence in monolayer and bilayer $\mathrm{WSe}_{2}$
}

\author{
Victor Funk, ${ }^{1}$ Koloman Wagner $\odot,{ }^{2}$ Edith Wietek $\odot,{ }^{2}$ Jonas D. Ziegler, ${ }^{2}$ Jonathan Förste, ${ }^{1}$ Jessica Lindlau, ${ }^{1}$ Michael Förg, ${ }^{1}$ \\ Kenji Watanabe $\odot{ }^{3}{ }^{3}$ Takashi Taniguchi, ${ }^{4}$ Alexey Chernikov, ${ }^{2,5, *}$ and Alexander Högele ${ }^{10}{ }^{1,6, \dagger}$ \\ ${ }^{1}$ Fakultät für Physik, Munich Quantum Center, and Center for NanoScience (CeNS), Ludwig-Maximilians-Universität München, \\ Geschwister-Scholl-Platz 1, 80539 München, Germany \\ ${ }^{2}$ Departement of Physics, University of Regensburg, 93053 Regensburg, Germany \\ ${ }^{3}$ Research Center for Functional Materials, National Institute for Materials Science, Tsukuba 305-0044, Japan \\ ${ }^{4}$ International Center for Materials Nanoarchitectonics, National Institute for Materials Science, Tsukuba 305-0044, Japan \\ ${ }^{5}$ Dresden Integrated Center for Applied Physics and Photonic Materials (IAPP) and Würzburg-Dresden Cluster of Excellence ct.qmat, \\ Technische Universität Dresden, 01062 Dresden, Germany \\ ${ }^{6}$ Munich Center for Quantum Science and Technology (MCQST), Schellingtraße 4, 80799 München, Germany
}

(Received 21 May 2021; revised 28 September 2021; accepted 30 September 2021; published 5 November 2021)

\begin{abstract}
We report an experimental study of temperature-dependent spectral line shapes of phonon sideband emission stemming from dark excitons in monolayer and bilayer $\mathrm{WSe}_{2}$. Using photoluminescence spectroscopy in the range from 4 to $100 \mathrm{~K}$, we observe a pronounced asymmetry in the phonon-assisted luminescence from momentum-indirect exciton reservoirs. We demonstrate that the corresponding spectral profiles are distinct from those of bright excitons with direct radiative decay pathways. The line-shape asymmetry reflects thermal distribution of exciton states with finite center-of-mass momenta, characteristic for phonon sideband emission. The extracted temperature of the exciton reservoirs is found to generally follow that of the crystal lattice, with deviations reflecting overheated populations. The latter are most pronounced in the bilayer case and at lowest temperatures. Our results add to the understanding of phonon-assisted recombination of momentum-dark excitons and, more generally, establish means to access the thermal distribution of finite-momentum excitons in atomically thin semiconductors with indirect band gaps.
\end{abstract}

DOI: 10.1103/PhysRevResearch.3.L042019

The nature of radiative electron-hole transitions has a rich history in semiconductor physics. Already from the early studies of germanium and silicon it became apparent that the requirements of simultaneous energy and momentum conservation fundamentally determine the optical response of semiconductors in both absorption [1,2] and emission [3-5]. Spectroscopy thus provides convenient and powerful means for discriminating between momentum-direct and momentum-indirect transitions, as well as for identifying interface- and phonon-assisted recombination [6,7]. Related methods have been successfully applied to two-dimensional van der Waals semiconductors to identify the prominent indirect-to-direct crossover from the bulk to the monolayer (ML) limit of transition metal dichalcogenides (TMDs) [8,9]. TMDs offer a particularly suitable platform to study the physics of momentum-direct and indirect electron-hole recombination processes in one material system. In contrast to conventional semiconductors, their properties are strongly

\footnotetext{
*alexey.chernikov@tu-dresden.de

$\dagger$ alexander.hoegele@1mu.de
}

Published by the American Physical Society under the terms of the Creative Commons Attribution 4.0 International license. Further distribution of this work must maintain attribution to the author(s) and the published article's title, journal citation, and DOI. affected by strong Coulomb interactions, leading to the formation of tightly bound exciton quasiparticles [10] with intriguing spin-valley physics resulting from the underlying band structure [11]. Following recent advances in sample preparation and doping control [12-16], TMDs exhibit a rich spectrum of exciton photoluminescence (PL) associated with electron-hole pairs from different high-symmetry points of the first Brillouin zone.

The PL of charge-neutral few-layer TMDs can be generally grouped into features of excitons with zero center-of-mass momentum $[8,9,17,18]$ and phonon sidebands of dark excitons with finite momenta [19-22]. The former decay radiatively with in- or out-of-plane polarization, resulting in characteristically symmetric spectral profiles in PL. Contrary to that, the latter originate from higher-order processes that require the assistance of phonons to conserve momentum upon photon emission. In the specific case of $\mathrm{WSe}_{2}$, phonon sidebands enrich the ML emission spectra by adding peaks energetically below the bright exciton X [17-19,2228]. The respective lower-energy PL features stem from $K K^{\prime}$ and $K Q$ excitons and correspond to phonon-assisted recombinations between valence band vacancies in the $K$ valley and conduction band electrons in the $K^{\prime}$ or $Q$ valley, respectively. In $\mathrm{WSe}_{2}$ bilayers (BLs), the energy of zero-momentum $K K$ excitons remains energetically close to $\mathrm{X}$ of the $\mathrm{ML}$, whereas the energy of $K Q$ excitons is substantially reduced due to interlayer hybridization effects $[8,9]$. As a result, the $K Q$ exciton reservoirs host the majority of the photoexcited 
exciton population at low temperatures and thus dominate the cryogenic PL spectra of $\mathrm{BL} \mathrm{WSe}_{2}[20,28,29]$.

To date, momentum-dark excitons and their phonon sidebands in $\mathrm{WSe}_{2}$ have been considered in the context of PL yield and dynamics [30-36] rather than in terms of spectral line-shape analysis. In particular, considerations regarding the spectral form of the emission that should reflect the characteristics of excitons with finite momenta [21,37] have been largely neglected so far despite the fact that phonon-assisted emission would allow for direct optical access to the exciton distribution beyond the restrictive limit of the radiative cone that otherwise applies for zero-phonon transitions. In the present Letter, we address these topics and report temperaturedependent studies of profound differences in the line shapes of PL peaks characteristic for direct and phonon-assisted transitions in $\mathrm{ML}$ and $\mathrm{BL} \mathrm{WSe} 2$. Our analysis demonstrates that asymmetric phonon sidebands are indeed captured by accounting for the thermal exciton distribution. In addition, we show that while the resulting effective exciton temperature generally follows that of the lattice, there are also notable deviations due to overheated exciton populations particularly pronounced in the BL case.

The samples studied in this Letter are based on exfoliated van der Waals heterostructures consisting of ML (samples A and B) and $\mathrm{BL}$ (also on sample B) $\mathrm{WSe}_{2}$ (HQ Graphene) encapsulated between hexagonal boron nitride $(\mathrm{hBN})$ layers and deposited on $\mathrm{SiO}_{2} / \mathrm{Si}$ substrate by polymer-assisted alldry viscoelastic stamping $[38,39]$. Subsequent annealing was carried out to enhance interlayer contact and reduce trapped residues [40]. Sample A was mounted in a continuousflow cryostat in a confocal microscope and excited by a continuous-wave (cw) laser at $2.33 \mathrm{eV}$ with a power of $500 \mathrm{nW}$ into an optical spot of $1 \mu \mathrm{m}$ diameter. The PL was dispersed with a spectrometer and detected by a chargecoupled-device camera.

In the ML PL of sample A, shown in Fig. 1(a) in the low-temperature range, the peak at $1.728 \mathrm{eV}$ stems from the neutral exciton $\mathrm{X}$, followed by the weaker trion doublet, $\mathrm{X}_{1}^{-}$ and $\mathrm{X}_{2}^{-}$, with $6 \mathrm{meV}$ exchange splitting [24]. The doublet is present due to residual $n$-doping of the sample. Between the doublet peaks, the $M_{1}$ peak at $30 \mathrm{meV}$ redshift from $\mathrm{X}$ has a rich history of controversial assignments, including a defectactivated recombination of $K K^{\prime}$ momentum-dark intervalley excitons [22,27], phonon-assisted emission from virtual trions [41], and a phonon sideband of momentum-dark $K Q$ excitons $[19,28]$. A series of redshifted peaks below the spin-dark $K K$ exciton (D) [17,42-44], labeled by an increasing subscript number with decreasing peak energy as $\mathrm{M}_{2}$ through $\mathrm{M}_{4}$, represent phonon sideband luminescence from long-lived dark excitons. In charge-neutral samples, recent work has ascribed the peaks $M_{2}$ and $M_{3}$ at 51 and $59 \mathrm{meV}$ redshifts from $X$ to phonon sidebands of momentum-dark $K K^{\prime}$ excitons [22,27]. However, it has also been shown that excitons from $K Q$ should exhibit phonon-assisted emission around $\mathrm{M}_{2}$ [21,36]. Finally, the $\mathrm{M}_{4}$ peak with $65 \mathrm{meV}$ redshift from $\mathrm{X}$ corresponds to the chiral-phonon replica of long-lived spin-dark D excitons $[22,26,27,45]$.

Sample B with data in Figs. 1(b) and 1(c) was studied in a closed-cycle magnetocryostat with a base temperature of $3.2 \mathrm{~K}$. Charge-doping in ML and BL was controlled via
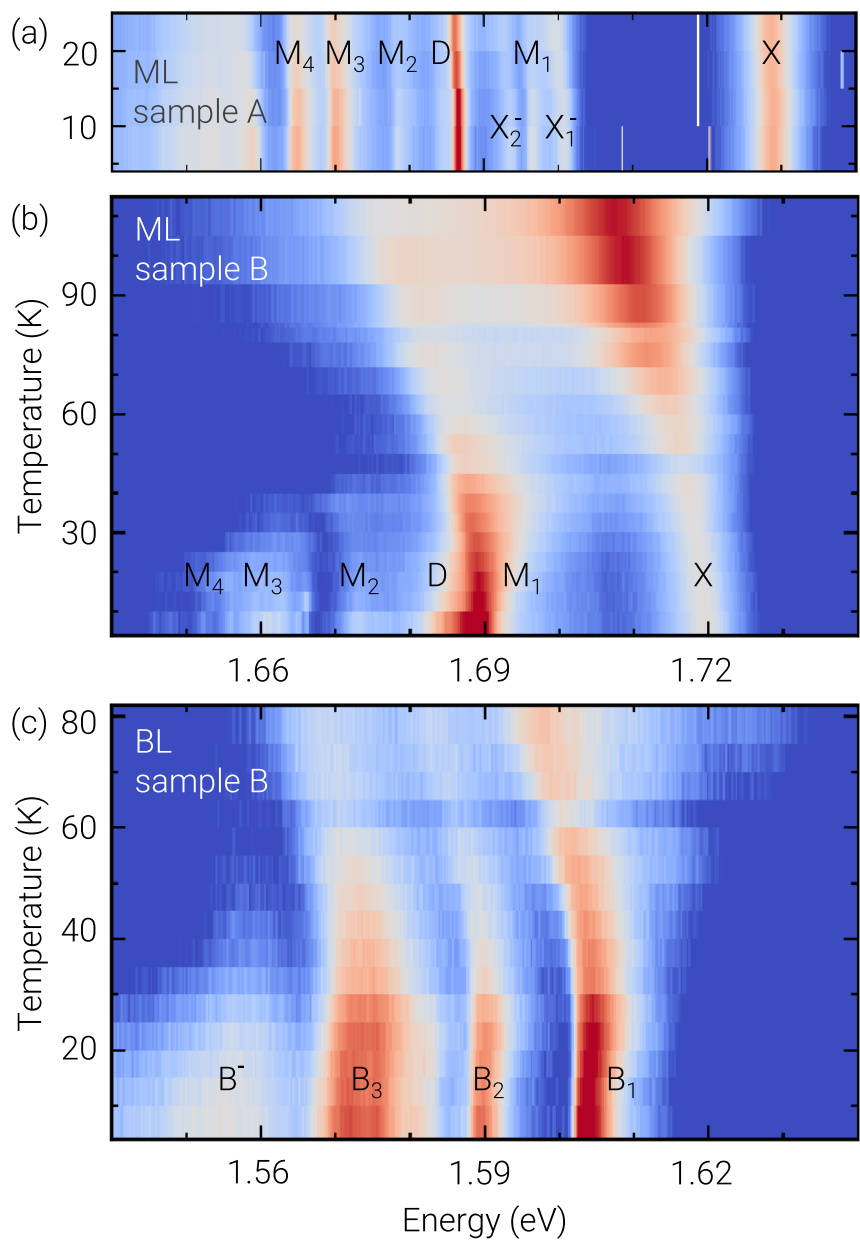

FIG. 1. (a), (b) Logarithmic false-color plots of the PL from samples $\mathrm{A}$ and $\mathrm{B}$ of $\mathrm{ML} \mathrm{WSe}_{2}$ encapsulated in $\mathrm{hBN}$, where the former exhibits features of residual doping and the latter is tuned to charge neutrality. (c) $\mathrm{PL}$ of $\mathrm{BL} \mathrm{WSe}_{2}$ from sample $\mathrm{B}$ tuned close to charge neutrality with a marginal contribution of the negative bilayer trion $\mathrm{B}^{-}$. Neutral spin-bright and spin-dark $K K$ exciton peaks are denoted as $\mathrm{X}$ and $\mathrm{D}$, and the trion doublet as $\mathrm{X}_{1}^{-}$and $\mathrm{X}_{2}^{-}$. The remaining $\mathrm{ML}$ and BL peaks are labeled by an increasing subscript number $n$ as $\mathrm{M}_{n}$ and $\mathrm{B}_{n}$, respectively.

the field effect by grounding a gold electrode in contact with the TMD crystal and applying a gate voltage with respect to the highly doped silicon substrate [28]. The sample was excited with a cw diode laser at $1.95 \mathrm{eV}$ at a low pump density in the linear regime. The temperature-dependent PL of a ML region on sample $B$ tuned to charge neutrality is shown in Fig. 1(b). At $3.2 \mathrm{~K}$, the PL characteristics are equivalent to those of sample A in Fig. 1(a) with an overall samplespecific redshift of $8 \mathrm{meV}$, fully suppressed trion features, and a larger inhomogeneous line broadening. For all peaks, a characteristic temperature-dependent redshift was observed with increasing temperature [23]. In addition, the PL intensity of the bright exciton $\mathrm{X}$ increased with temperature at the expense of all lower-lying peaks. This is consistent with a temperature-dependent population redistribution from lower dark to energetically higher bright exciton states, in agreement with previous experimental $[42,44]$ and more recent theoretical studies [21]. 
(a)
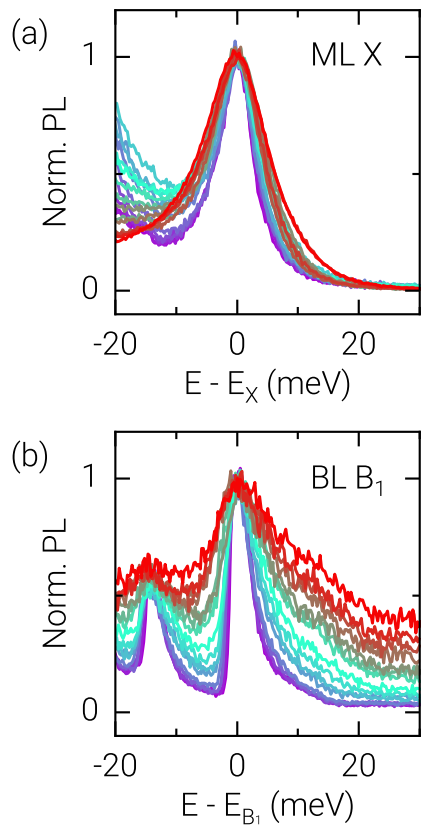
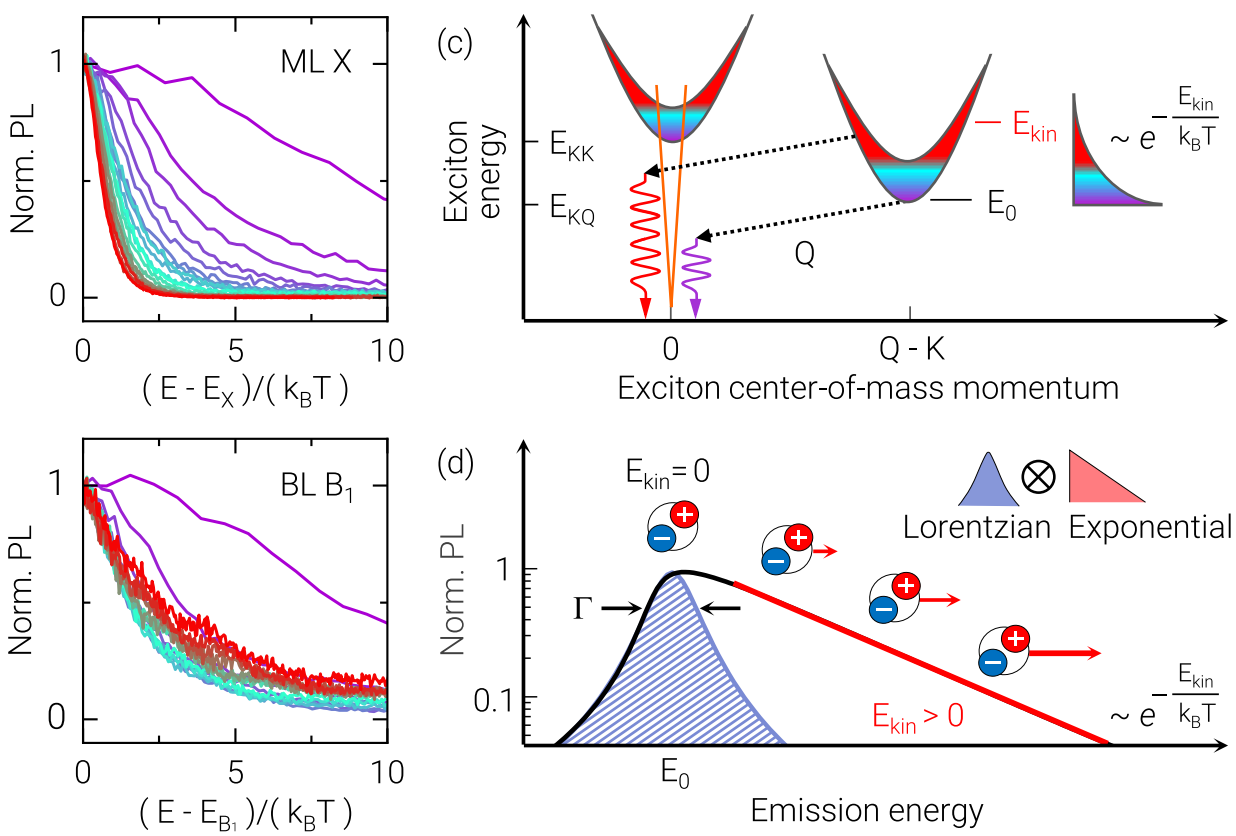

FIG. 2. (a) Left panel: PL spectra of ML in sample B at temperatures from 4 to $120 \mathrm{~K}$ (colored from purple to red), normalized to unity and shifted horizontally to the energy $E_{X}$ of the bright exciton peak X at $4 \mathrm{~K}$. Right panel: High-energy shoulder of the spectra with energy axis normalized by $k_{B} T$. (b) Same for BL in the temperature range from 4 to $80 \mathrm{~K}$ with the energy of the peak $\mathrm{B}_{1}$ as reference. (c) Schematic representation of direct and indirect decay processes for excitons. The center-of-mass momenta of $K K$ excitons, such as $\mathrm{X}$ or $\mathrm{D}$, with zerophonon radiative decay are restricted to cold excitons (blue shaded population inside the orange light cone). In contrast, phonon sideband luminescence of momentum-dark excitons such as $K Q$ with energy minimum $E_{0}=E_{K Q}$ and exciton center-of-mass momentum $Q-K$ is assisted by phonons with momentum $Q$ and thus also includes emission from hot excitons (thermally activated red shaded population). The thermal Boltzmann occupancy of exciton states with finite kinetic energy $E_{\mathrm{kin}}>E_{0}$ above their respective dispersion minimum is represented by the area of colored shades. (d) Illustration of the phonon sideband asymmetry: The homogeneously broadened Lorentzian spectrum with linewidth $\Gamma$ is convoluted on the high-energy side with the contribution from excitons with finite kinetic energy $E_{\text {kin }}>0$ according to their thermal occupancy in Boltzmann approximation.

Complementary to the ML results presented in Figs. 1(a) and 1(b), temperature-dependent PL from encapsulated BL $\mathrm{WSe}_{2}$ tuned close to charge neutrality is shown in Fig. 1(c). It features a multipeak structure labeled by an increasing subscript number with decreasing peak energy as $\mathrm{B}_{1}$ through $\mathrm{B}_{3}$. These peaks, located about 110 to $150 \mathrm{meV}$ below the ML peak X, correspond to phonon sidebands of momentumindirect $K Q$ excitons $[20,28,29]$. Due to a marginal departure from the charge neutrality condition, the lowest-energy peak $\mathrm{B}^{-}$is weakly present as luminescence from bilayer $K Q$ trions [20] with the excess electrons accommodated at the conduction band edge by the $Q$ valleys.

Interestingly, whereas the temperature-induced energy shifts are similar for the BL case, the evolution of the PL intensity with increasing temperature differs significantly from the ML. This difference is closely related to the indirect band gap of the BL that is substantially smaller than the energy of the direct $K K$ transition. The thermal energy on the order of $10 \mathrm{meV}$ accessed in our experiments is far from sufficient to provide substantial thermal excitation of the population from the momentum-dark $K Q$ reservoir into bright $K K$ states with efficient radiative decay pathways. Therefore, the relative intensities of all peaks remain largely the same with a moderate decrease of the total emission signal. Only $\mathrm{B}^{-}$quenches strongly, as expected for a weakly bound trion state.
Following the observation and identification of the relevant transitions, we now focus on their characteristic spectral line shapes. In the left panels of Fig. 2(a) and 2(b), the spectra recorded at different temperatures were normalized and shifted to the energy of $X$ and $B_{1}$ for ML and BL, respectively. Direct comparison highlights the main difference in their spectral profiles and the associated temperature dependence. Whereas the ML bright exciton peak X is well described by a symmetric peak function such as a Lorentzian, the BL peaks are asymmetric with a pronounced high-energy shoulder (similar asymmetry is also found for the ML peaks $\mathrm{M}_{1}$ through $\mathrm{M}_{4}$ as discussed below). Their temperature dependence also differs from that of the bright exciton $\mathrm{X}$, which broadens thermally with increasing temperature and remains symmetric while the asymmetry of the BL peaks becomes increasingly prominent.

Clearly, the scenario of linewidth broadening due to exciton-phonon scattering applicable to $X[23,31]$ is insufficient to explain both the presence and the temperaturedependent increase of the higher-energy shoulder of BL peaks. As opposed to the direct radiative decay pathway of $\mathrm{X}$, recombination of momentum-indirect excitons requires both a photon and a phonon to simultaneously satisfy energy and momentum conservation constraints. Since an arbitrary crystal momentum can be transferred to a phonon, all excitons, including those with finite center-of-mass momenta, 
can recombine via phonon-assisted pathways indicated in the schematics of Fig. 2(c). In consequence, the thermal distribution of exciton momenta directly manifests in the characteristic high-energy shoulder of the resulting PL spectra as illustrated in Fig. 2(d) and observed earlier on conventional semiconductors $[37,46,47]$. This is in stark contrast to direct exciton recombination (also known as zero-phonon line) where only excitons with vanishing center-of-mass momenta within the radiative cone can recombine. This difference becomes even more apparent by rescaling the energy axis at each temperature by the thermal energy of the lattice $k_{B} T$, with the high-energy shoulder shown in the right panels of Figs. 2(a) and 2(b). In this representation, the PL spectra of $\mathrm{X}$ are all distinct due to symmetric thermal broadening. On the contrary, the BL spectra collapse onto a universal curve for all temperatures above $10 \mathrm{~K}$, when the thermally activated high-energy flank dominates over symmetric broadening.

To analyze these observations quantitatively, we model the phonon sidebands by Lorentzians weighted with the thermal occupation of exciton states with finite kinetic energies $E_{\text {kin }}=$ $E-E_{0}>0$, as illustrated in Fig. 2(d). This approach yields the spectral line shape $I(E)$ of phonon sideband resonances at temperature $T$ as

$$
I(E)=I_{0} \int_{0}^{\infty} \frac{\Gamma / 2}{\left(E-E_{0}\right)^{2}+(\Gamma / 2)^{2}} \exp \left(-\frac{E_{\text {kin }}}{k_{B} T}\right) d E,
$$

where $I_{0}$ is a normalization constant, $\Gamma$ is the full-width at half-maximum (FWHM) Lorentzian linewidth in units of energy, and $k_{B}$ is the Boltzmann constant. The exponential term in the integral corresponds to the Boltzmann occupation of exciton states with kinetic energy measured with respect to the corresponding exciton band minimum at $E_{0}$ [e.g., as in Fig. 2(c) where $\left.E_{0}=E_{K Q}\right]$. This expression for the spectral line shape, equivalent to the result of a rigorous derivation in Ref. [21], was used to model the experimental spectra at different temperatures using $I_{0}, E_{0}, \Gamma$, and the effective exciton temperature $T=T_{\text {exc }}$ as fit parameters. We also note that the Boltzmann approximation for bosonic exciton quasiparticles should be reasonable due to rather low population densities in our experiments.

The results of least-squares numerical fits to the spectra of selected ML and BL peaks in samples A and B, respectively, are shown as solid lines in Fig. 3(a)-3(c). The ML and BL spectra were shifted at each temperature to the energy of the $\mathrm{X}$ and $\mathrm{B}_{1}$ peaks, respectively, normalized and offset for clarity. The overall good fit quality over the studied temperature range shows that peaks $\mathrm{M}_{2}, \mathrm{M}_{3}$, and $\mathrm{M}_{4}$ in Fig. 3(a) (note that the peaks $\mathrm{M}_{3}$ and $\mathrm{M}_{4}$ of sample $A$ were used for a similar analysis in the context of exciton diffusion [48]), $\mathrm{M}_{1}$ in Fig. 3(b) as well as $B_{1}$ and $B_{2}$ in Fig. 3(c) are all asymmetrically broadened and adequately modeled by Eq. (1). In particular, it demonstrates the necessity to convolute a thermally broadened Lorentzian with a high-energy shoulder of thermal exciton occupation to account for the experimental observations.

In the progress of our analysis, we first confirm that the lattice temperature corresponds to the externally controlled temperature. To this end, we plot in Fig. 4(a) the energy $E_{0}$ obtained from best fits to the peaks $\mathrm{X}, \mathrm{B}_{1}$ and $\mathrm{B}_{2}$ in sample $\mathrm{B}$ as a function of temperature. The evolution shows for all peaks the expected redshift with increasing temperature and

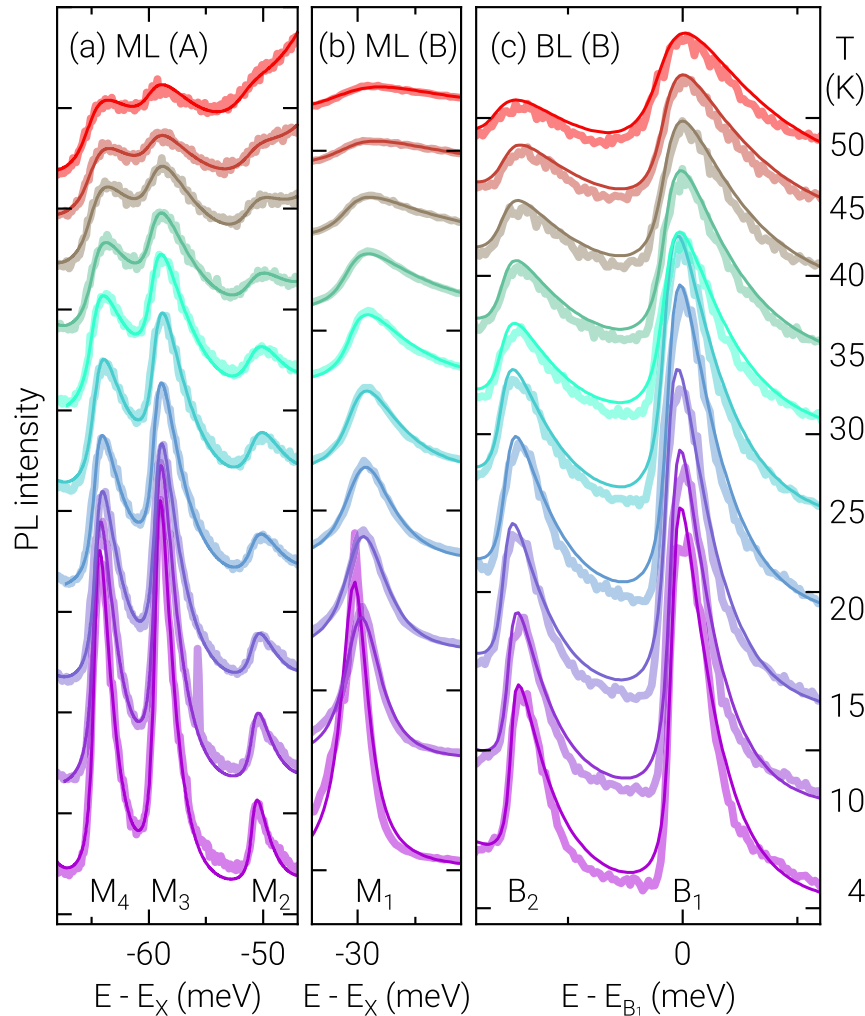

FIG. 3. Model fits of asymmetric peaks in (a) and (b) for MLs of sample A and B, respectively, and (c) BL of sample B. The fits (solid lines) to the spectra for temperatures ranging from 4 to $50 \mathrm{~K}$ were obtained according to Eq. (1) with exciton temperature, Lorentzian linewidth, peak energy, and intensity as free fit parameters. Note the pronounced asymmetry on the high-energy side of all ML and BL peaks and the good agreement with the model of contributions from thermally activated excitons with finite center-of-mass momenta.

substantial contributions from band-gap renormalization by thermally activated phonons. Using the relation [49]

$$
E_{0}(T)=E_{0}^{0}-S \hbar \bar{\Omega}\left[\operatorname{coth}\left(\frac{\hbar \bar{\Omega}}{2 k_{B} T}\right)-1\right],
$$

we model the thermal energy dispersions in Fig. 4(a) with the zero temperature limit of the peak energy $E_{0}^{0}$ as a free fit parameter and use the values for the coupling constant $S$ (2.06 and 1.75 in ML and BL) and the average phonon energy $\hbar \bar{\Omega}=15 \mathrm{meV}$ determined previously in a much wider temperature range [23]. The quantitative agreement with the observed shifts in our samples strongly supports a one-to-one correspondence between the experimental temperature and the temperature of the crystal lattice.

The second temperature-dependent effect is the symmetric spectral broadening by pure dephasing, represented by the Lorentzian linewidth parameter $\Gamma$ in Eq. (1). The resulting temperature dependence shown in Fig. 4(b) is known to stem from thermal broadening by both linear-acoustic and fixed-energy phonons. The latter can generally include both optical and high-momentum acoustic modes in TMD materials $[23,31,50,51]$. The approximate expression commonly used for quantitative assessment relates the spectral broadening to the phonon occupation weighted by their interaction 

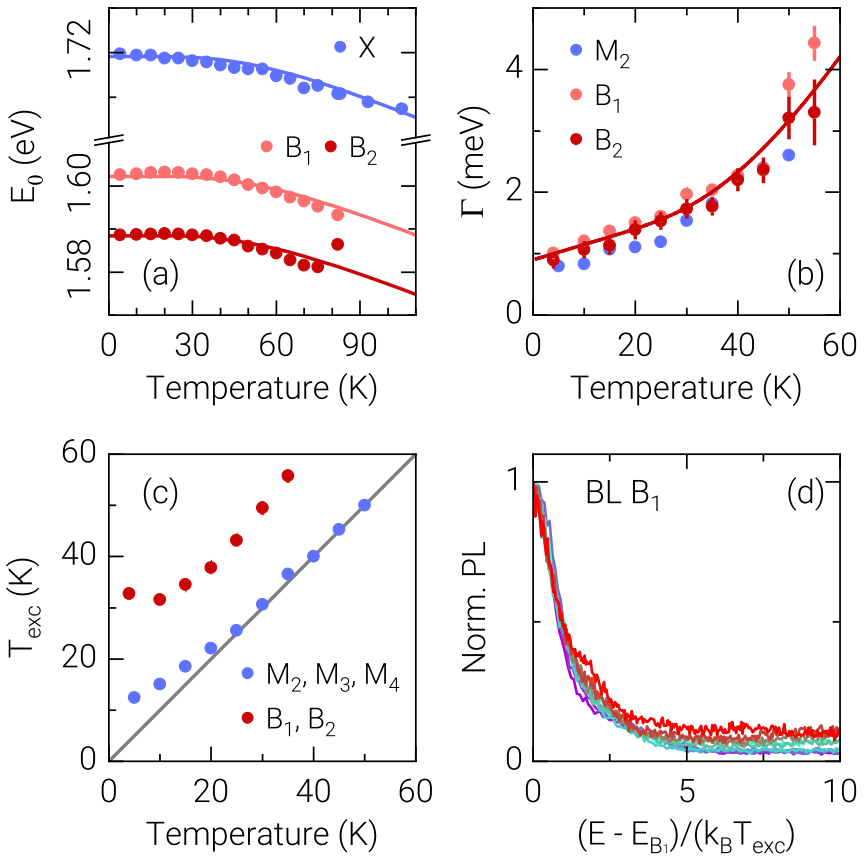

FIG. 4. Least-squares parameters obtained from model fits. (a) Evolution of the peak energy with temperature for $\mathrm{X}$ in $\mathrm{ML}$ as well as for $\mathrm{B}_{1}$ and $\mathrm{B}_{2}$ in $\mathrm{BL}$ of sample $\mathrm{B}$. The solid lines show best fits to thermal band-gap renormalization according to Eq. (2) with parameters from Ref. [23]. (b) Lorentzian linewidth of the ML peak $\mathrm{M}_{2}$ in sample $\mathrm{A}$ and $\mathrm{BL}$ peaks $\mathrm{B}_{1}$ and $\mathrm{B}_{2}$ in sample $\mathrm{B}$. The solid line shows best fit for BL peaks taking into account thermal phonon broadening according to Eq. (3) (c) Effective exciton temperature $T_{\text {exc }}$ obtained from best fits to temperature-dependent spectra with Eq. (1) and plotted versus the nominal sample temperature (the solid grey line shows the lattice temperature; $\mathrm{ML}$ and $\mathrm{BL}$ peaks from sample A and B, respectively). (d) Blue shoulder of $B_{1}$ with energy axis normalized by the effective exciton temperature $k_{B} T_{\text {exc }}$ for direct comparison with the right panel of Fig. 2(b). Note the universality of the high-energy shoulder asymmetry arising from the phonon sideband emission by thermally activated excitons.

strength with electron-hole pairs [52]:

$$
\Gamma(T)=\Gamma_{0}-a T+b\left[\exp \left(\frac{\hbar \Omega}{k T}\right)-1\right]^{-1} .
$$

Here $\Gamma_{0}$ represents the zero temperature limit (excluding inhomogeneity) and $a$ and $b$ denote the coupling constants for linear-acoustic and fixed-energy phonons, respectively. The energy of the dominant phonon in the last term responsible for a superlinear linewidth dependence on the temperature is denoted by $\hbar \Omega$.

The best unconstrained fit, shown as the red solid line in Fig. 4(b), was obtained with $\Gamma_{0}=0.9 \pm 0.2 \mathrm{meV}, a=25 \pm$ $10 \mu \mathrm{eV} / \mathrm{K}, b=32 \pm 17 \mathrm{meV}$, and $\hbar \Omega=15 \mathrm{meV}$ (constraining $\hbar \Omega$ to the energy of the LO phonon $31.25 \mathrm{meV}$ instead yields a rather poor match to the data). This characteristic phonon energy is consistent with $16.3 \mathrm{meV}$ determined from coherent nonlinear spectroscopy for $\mathrm{ML} \mathrm{WSe}_{2}$ [50] and close to the zone-edge phonon energy of $20 \mathrm{meV}$ in related studies of $\mathrm{WS}_{2}$ [31]. The coupling constant $a$ obtained from the above analysis is within the range of previously reported values of $20-60 \mu \mathrm{eV} / \mathrm{K}$ for $\mathrm{WSe}_{2}$ MLs [30,53,54].
As the last fit parameter, we discuss the effective exciton temperature $T_{\text {exc }}$ plotted against the nominal experimental temperature in Fig. 4(c). From our fit procedure according to Eq. (1), we find that the extracted exciton temperature generally follows that of the lattice. The agreement is very close for ML peaks in particular, as illustrated by the gray solid line in Fig. 4(c) showing the lattice temperature calibrated earlier. In contrast, the momentum-indirect excitons in the $\mathrm{BL}$ are overheated by about $20 \mathrm{~K}$ with respect to the lattice temperature.

This observation is indicative of vastly different timescales of the exciton lifetimes in the studied samples. In the ML case, the phonon sidebands $\mathrm{M}_{2}, \mathrm{M}_{3}$, and $\mathrm{M}_{4}$ stem from dark excitons that decay on the order of several 100's of ps. As a consequence, they live sufficiently long to ensure cooling of hot excitons on a timescale of a few 10's of ps [36]. Similar arguments apply to the peak $\mathrm{M}_{1}$ without unambiguous discrimination by the exciton $g$ factor $[22,27,28]$, be it related to direct decay of momentum-indirect $K K^{\prime}$ excitons [22,27] or a phonon sideband of the $K Q$ reservoir [19]. In contrast, the lifetime of the lowest-energy $K Q$ exciton reservoir in $\mathrm{BL}$ with phonon sidebands $\mathrm{B}_{1}$ through $\mathrm{B}_{3}$ is only $25 \mathrm{ps}$ [55]. This difference of at least one order of magnitude in the momentum-dark exciton lifetimes in ML and BL, paired with a higher relative injection energy for the latter, accounts for overheating of momentum-dark $K Q$ excitons in the BL case. This fact is also reflected by the departure from one universal spectrum in the right panel of Fig. 2(b). If, however, the blue shoulder of $\mathrm{B}_{1}$ is rescaled by $k_{B} T_{\text {exc }}$ as in Fig. 4(d), we recover the expected notion that phonon sidebands reflect the thermal Boltzmann occupation of exciton states according to their kinetic energy and temperature.

In conclusion, we experimentally identify characteristic spectral line-shape asymmetry of phonon sidebands in the PL of $\mathrm{ML}$ and $\mathrm{BL} \mathrm{WSe}_{2}$. In contrast to symmetric PL peaks from zero-momentum excitons, the asymmetric profiles arise from thermal distribution of excitons in long-lived dark reservoirs with finite center-of-mass momenta. The extracted exciton temperature is found to generally follow that of the crystal lattice. However, we also find deviations that are indicative of the presence of overheated exciton populations in the system. These are mostly pronounced at lowest temperatures and in the BL case, when the recombination lifetime approaches the timescale of the cooling processes. Such direct experimental access to the temperature of excitons with finite center-of-mass momenta provides additional insight into the fundamental electron-hole recombination processes in atomically thin semiconductors. For the entire class of TMD semiconductors and heterostructures, our work emphasizes the distinctions of momentum-direct and momentum-indirect recombination pathways, identifying spectroscopic means of distinguishing the respective exciton reservoirs and highlighting the role of the phonon-assisted emission for long-lived excitons.

The authors thank Ermin Malic and members of his group for helpful discussions. This research was funded by the European Research Council (ERC) under Grant Agreement No. 772195 as well as the Deutsche Forschungsgemeinschaft (DFG, German Research Foundation) within the Priority 
Programme SPP 2244 2DMP (Project No. 443405595), Emmy Noether Initiative (CH1672/1, Project No. 287022282), SFB 1244 (Project No. B05), and the Germany's Excellence Strategy Munich Center for Quantum Science and Technology (MCQST) (EXC 2111, Project-ID 390814868) and Würzburg-Dresden Cluster of Excellence on Complexity and Topology in Quantum Matter (ct.qmat)
(EXC 2147, Project-ID 390858490). A.H. acknowledges support from the Center for NanoScience (CeNS) and the LMUinnovativ project Functional Nanosystems (FuNS). K.W. and T.T. acknowledge support from the Elemental Strategy Initiative conducted by the MEXT, Japan, Grant No. JPMXP0112101001, JSPS KAKENHI Grant No. JP20H00354 and the CREST (No. JPMJCR15F3), JST.
[1] G. G. Macfarlane, T. P. McLean, J. E. Quarrington, and V. Roberts, Fine structure in the absorption-edge spectrum of Ge, Phys. Rev. 108, 1377 (1957).

[2] G. G. Macfarlane, T. P. McLean, J. E. Quarrington, and V. Roberts, Fine structure in the absorption-edge spectrum of $\mathrm{Si}$, Phys. Rev. 111, 1245 (1958).

[3] R. Newman, Optical studies of injected carriers. II. Recombination radiation in germanium, Phys. Rev. 91, 1313 (1953).

[4] J. R. Haynes, New radiation resulting from recombination of holes and electrons in germanium, Phys. Rev. 98, 1866 (1955).

[5] J. R. Haynes and W. C. Westphal, Radiation resulting from recombination of holes and electrons in silicon, Phys. Rev. 101, 1676 (1956).

[6] M. Bonfanti, E. Grilli, M. Guzzi, M. Virgilio, G. Grosso, D. Chrastina, G. Isella, H. von Känel, and A. Neels, Optical transitions in $\mathrm{Ge} / \mathrm{SiGe}$ multiple quantum wells with Ge-rich barriers, Phys. Rev. B 78, 041407(R) (2008).

[7] A. Giorgioni, E. Gatti, E. Grilli, A. Chernikov, S. Chatterjee, D. Chrastina, G. Isella, and M. Guzzi, Photoluminescence decay of direct and indirect transitions in $\mathrm{Ge} / \mathrm{SiGe}$ multiple quantum wells, J. Appl. Phys. 111, 013501 (2012).

[8] K. F. Mak, C. Lee, J. Hone, J. Shan, and T. F. Heinz, Atomically Thin $\mathrm{MoS}_{2}$ : A New Direct-Gap Semiconductor, Phys. Rev. Lett. 105, 136805 (2010).

[9] A. Splendiani, L. Sun, Y. Zhang, T. Li, J. Kim, C.-Y. Chim, G. Galli, and F. Wang, Emerging photoluminescence in monolayer $\mathrm{MoS}_{2}$, Nano Lett. 10, 1271 (2010).

[10] G. Wang, A. Chernikov, M. M. Glazov, T. F. Heinz, X. Marie, T. Amand, and B. Urbaszek, Colloquium: Excitons in atomically thin transition metal dichalcogenides, Rev. Mod. Phys. 90, 021001 (2018).

[11] X. Xu, W. Yao, D. Xiao, and T. F. Heinz, Spin and pseudospins in layered transition metal dichalcogenides, Nat. Phys. 10, 343 (2014).

[12] K. F. Mak, K. He, C. Lee, G. H. Lee, J. Hone, T. F. Heinz, and J. Shan, Tightly bound trions in monolayer $\mathrm{MoS}_{2}$, Nat. Mater. 12, 207 (2013).

[13] J. S. Ross, S. Wu, H. Yu, N. J. Ghimire, A. M. Jones, G. Aivazian, J. Yan, D. G. Mandrus, D. Xiao, W. Yao, and X. $\mathrm{Xu}$, Electrical control of neutral and charged excitons in a monolayer semiconductor, Nat. Commun. 4, 1474 (2013).

[14] F. Cadiz, E. Courtade, C. Robert, G. Wang, Y. Shen, H. Cai, T. Taniguchi, K. Watanabe, H. Carrere, D. Lagarde, M. Manca, T. Amand, P. Renucci, S. Tongay, X. Marie, and B. Urbaszek, Excitonic Linewidth Approaching the Homogeneous Limit in $\mathrm{MoS}_{2}$-Based Van Der Waals Heterostructures, Phys. Rev. X 7, 021026 (2017).

[15] O. A. Ajayi, J. V. Ardelean, G. D. Shepard, J. Wang, A. Antony, T. Taniguchi, K. Watanabe, T. F. Heinz, S. Strauf, X.-Y. Zhu, and J. C. Hone, Approaching the intrinsic photoluminescence linewidth in transition metal dichalcogenide monolayers, 2D Mater. 4, 031011 (2017).

[16] J. Wierzbowski, J. Klein, F. Sigger, C. Straubinger, M. Kremser, T. Taniguchi, K. Watanabe, U. Wurstbauer, A. W. Holleitner, M. Kaniber, K. Müller, and J. J. Finley, Direct exciton emission from atomically thin transition metal dichalcogenide heterostructures near the lifetime limit, Sci. Rep. 7, 12383 (2017).

[17] Y. Zhou, G. Scuri, D. S. Wild, A. A. High, A. Dibos, L. A. Jauregui, C. Shu, K. De Greve, K. Pistunova, A. Y. Joe, T. Taniguchi, K. Watanabe, P. Kim, M. D. Lukin, and H. Park, Probing dark excitons in atomically thin semiconductors via near-field coupling to surface plasmon polaritons, Nat. Nanotechnol. 12, 856 (2017).

[18] X.-X. Zhang, T. Cao, Z. Lu, Y.-C. Lin, F. Zhang, Y. Wang, Z. Li, J. C. Hone, J. A. Robinson, D. Smirnov, S. G. Louie, and T. F. Heinz, Magnetic brightening and control of dark excitons in monolayer $\mathrm{WSe}_{2}$, Nat. Nanotechnol. 12, 883 (2017).

[19] J. Lindlau, C. Robert, V. Funk, M. Förg, L. Colombier, A. Neumann, T. Taniguchi, K. Watanabe, M. M. Glazov, X. Marie, B. Urbaszek, and A. Högele, Identifying optical signatures of momentum-dark excitons in monolayer transition metal dichalcogenides, arXiv:1710.00988.

[20] J. Lindlau, M. Selig, A. Neumann, L. Colombier, J. Förste, V. Funk, M. Förg, J. Kim, G. Berghäuser, T. Taniguchi, K. Watanabe, F. Wang, E. Malic, and A. Högele, The role of momentum-dark excitons in the elementary optical response of bilayer $\mathrm{WSe}_{2}$, Nat. Commun. 9, 2586 (2018).

[21] S. Brem, A. Ekman, D. Christiansen, F. Katsch, M. Selig, C. Robert, X. Marie, B. Urbaszek, A. Knorr, and E. Malic, Phonon-assisted photoluminescence from indirect excitons in monolayers of transition-metal dichalcogenides, Nano Lett. 20, 2849 (2020).

[22] M. He, P. Rivera, D. Van Tuan, N. P. Wilson, M. Yang, T. Taniguchi, K. Watanabe, J. Yan, D. G. Mandrus, H. Yu, H. Dery, $\mathrm{W}$. Yao, and $\mathrm{X}$. Xu, Valley phonons and exciton complexes in a monolayer semiconductor, Nat. Commun. 11, 618 (2020).

[23] A. Arora, M. Koperski, K. Nogajewski, J. Marcus, C. Faugeras, and M. Potemski, Excitonic resonances in thin films of $\mathrm{WSe}_{2}$ : from monolayer to bulk material, Nanoscale 7, 10421 (2015).

[24] E. Courtade, M. Semina, M. Manca, M. M. Glazov, C. Robert, F. Cadiz, G. Wang, T. Taniguchi, K. Watanabe, M. Pierre, W. Escoffier, E. L. Ivchenko, P. Renucci, X. Marie, T. Amand, and B. Urbaszek, Charged excitons in monolayer $\mathrm{WSe}_{2}$ : Experiment and theory, Phys. Rev. B 96, 085302 (2017).

[25] M. Koperski, M. R. Molas, A. Arora, K. Nogajewski, A. O. Slobodeniuk, C. Faugeras, and M. Potemski, Optical properties 
of atomically thin transition metal dichalcogenides: Observations and puzzles, Nanophotonics 6, 1289 (2017).

[26] E. Liu, J. van Baren, T. Taniguchi, K. Watanabe, Y.-C. Chang, and C. H. Lui, Valley-selective chiral phonon replicas of dark excitons and trions in monolayer $\mathrm{WSe}_{2}$, Phys. Rev. Research $\mathbf{1}$, 032007(R) (2019).

[27] E. Liu, J. van Baren, C.-T. Liang, T. Taniguchi, K. Watanabe, N. M. Gabor, Y.-C. Chang, and C. H. Lui, Multipath Optical Recombination of Intervalley Dark Excitons and Trions in Monolayer $\mathrm{WSe}_{2}$, Phys. Rev. Lett. 124, 196802 (2020).

[28] J. Förste, N. V. Tepliakov, S. Y. Kruchinin, J. Lindlau, V. Funk, M. Förg, K. Watanabe, T. Taniguchi, A. S. Baimuratov, and A. Högele, Exciton g-factors in monolayer and bilayer $\mathrm{WSe}_{2}$ from experiment and theory, Nat. Commun. 11, 4539 (2020).

[29] O. B. Aslan, M. Deng, M. L. Brongersma, and T. F. Heinz, Strained bilayer $\mathrm{WSe}_{2}$ with reduced exciton-phonon coupling, Phys. Rev. B 101, 115305 (2020).

[30] G. Moody, C. Kavir Dass, K. Hao, C.-H. Chen, L.-J. Li, A. Singh, K. Tran, G. Clark, X. Xu, G. Berghäuser, E. Malic, A. Knorr, and $\mathrm{X}$. Li, Intrinsic homogeneous linewidth and broadening mechanisms of excitons in monolayer transition metal dichalcogenides, Nat. Commun. 6, 8315 (2015).

[31] M. Selig, G. Berghäuser, A. Raja, P. Nagler, C. Schüller, T. F. Heinz, T. Korn, A. Chernikov, E. Malic, and A. Knorr, Excitonic linewidth and coherence lifetime in monolayer transition metal dichalcogenides, Nat. Commun. 7, 13279 (2016).

[32] E. Malic, M. Selig, M. Feierabend, S. Brem, D. Christiansen, F. Wendler, A. Knorr, and G. Berghäuser, Dark excitons in transition metal dichalcogenides, Phys. Rev. Materials 2, 014002 (2018).

[33] M. Selig, G. Berghäuser, M. Richter, R. Bratschitsch, A. Knorr, and E. Malic, Dark and bright exciton formation, thermalization, and photoluminescence in monolayer transition metal dichalcogenides, 2D Mater. 5, 035017 (2018).

[34] G. Berghäuser, P. Steinleitner, P. Merkl, R. Huber, A. Knorr, and E. Malic, Mapping of the dark exciton landscape in transition metal dichalcogenides, Phys. Rev. B 98, 020301(R) (2018).

[35] G.-H. Peng, P.-Y. Lo, W.-H. Li, Y.-C. Huang, Y.-H. Chen, C.-H. Lee, C.-K. Yang, and S.-J. Cheng, Distinctive signatures of the spin- and momentum-forbidden dark exciton states in the photoluminescence of strained $\mathrm{WSe}_{2}$ monolayers under thermalization, Nano Lett. 19, 2299 (2019).

[36] R. Rosati, K. Wagner, S. Brem, R. Perea-Causín, E. Wietek, J. Zipfel, J. D. Ziegler, M. Selig, T. Taniguchi, K. Watanabe, A. Knorr, A. Chernikov, and E. Malic, Temporal evolution of low-temperature phonon sidebands in transition metal dichalcogenides, ACS Photonics 7, 2756 (2020).

[37] C. Klingshirn, Semiconductor Optics, 3rd ed. (Springer, Berlin, 2007).

[38] A. Castellanos-Gomez, M. Buscema, R. Molenaar, V. Singh, L. Janssen, H. S. Van Der Zant, and G. A. Steele, Deterministic transfer of two-dimensional materials by all-dry viscoelastic stamping, 2D Mater. 1, 011002 (2014).

[39] F. Pizzocchero, L. Gammelgaard, B. S. Jessen, J. M. Caridad, L. Wang, J. Hone, P. Bøggild, and T. J. Booth, The hot pick-up technique for batch assembly of van der Waals heterostructures, Nat. Commun. 7, 11894 (2016).

[40] A. Jain, P. Bharadwaj, S. Heeg, M. Parzefall, T. Taniguchi, K. Watanabe, and L. Novotny, Minimizing residues and strain in 2D materials transferred from PDMS, Nanotechnology 29, 265203 (2018).

[41] D. Van Tuan, A. M. Jones, M. Yang, X. Xu, and H. Dery, Virtual Trions in the Photoluminescence of Monolayer TransitionMetal Dichalcogenides, Phys. Rev. Lett. 122, 217401 (2019).

[42] X. X. Zhang, Y. You, S. Y. F. Zhao, and T. F. Heinz, Experimental Evidence for Dark Excitons in Monolayer WSe 2 , Phys. Rev. Lett. 115, 257403 (2015).

[43] G. Wang, C. Robert, M. M. Glazov, F. Cadiz, E. Courtade, T. Amand, D. Lagarde, T. Taniguchi, K. Watanabe, B. Urbaszek, and X. Marie, In-Plane Propagation of Light in Transition Metal Dichalcogenide Monolayers: Optical Selection Rules, Phys. Rev. Lett. 119, 047401 (2017).

[44] C. Robert, T. Amand, F. Cadiz, D. Lagarde, E. Courtade, M. Manca, T. Taniguchi, K. Watanabe, B. Urbaszek, and X. Marie, Fine structure and lifetime of dark excitons in transition metal dichalcogenide monolayers, Phys. Rev. B 96, 155423 (2017).

[45] Z. Li, T. Wang, C. Jin, Z. Lu, Z. Lian, Y. Meng, M. Blei, S. Gao, T. Taniguchi, K. Watanabe, T. Ren, S. Tongay, L. Yang, D. Smirnov, T. Cao, and S. F. Shi, Emerging photoluminescence from the dark-exciton phonon replica in monolayer $\mathrm{WSe}_{2}$, Nat. Commun. 10, 2469 (2019).

[46] M. Umlauff, J. Hoffmann, H. Kalt, W. Langbein, J. M. Hvam, M. Scholl, J. Söllner, M. Heuken, B. Jobst, and D. Hommel, Direct observation of free-exciton thermalization in quantumwell structures, Phys. Rev. B 57, 1390 (1998).

[47] S. J. Xu, G. Q. Li, S.-J. Xiong, and C. M. Che, Temperature dependence of the LO phonon sidebands in free exciton emission of GaN, J. Appl. Phys. 99, 073508 (2006).

[48] K. Wagner, J. Zipfel, R. Rosati, E. Wietek, J. D. Ziegler, S. Brem, R. Perea-Causín, T. Taniguchi, K. Watanabe, M. M. Glazov, E. Malic, and A. Chernikov, Nonclassical Exciton Diffusion in Monolayer WSe ${ }_{2}$, Phys. Rev. Lett. 127, 076801 (2021).

[49] K. P. O'Donnell and X. Chen, Temperature dependence of semiconductor band gaps, Appl. Phys. Lett. 58, 2924 (1991).

[50] P. Dey, J. Paul, Z. Wang, C. E. Stevens, C. Liu, A. H. Romero, J. Shan, D. J. Hilton, and D. Karaiskaj, Optical Coherence in Atomic-Monolayer Transition-Metal Dichalcogenides Limited by Electron-Phonon Interactions, Phys. Rev. Lett. 116, 127402 (2016).

[51] S. Shree, M. Semina, C. Robert, B. Han, T. Amand, A. Balocchi, M. Manca, E. Courtade, X. Marie, T. Taniguchi, K. Watanabe, M. M. Glazov, and B. Urbaszek, Observation of exciton-phonon coupling in $\mathrm{MoSe}_{2}$ monolayers, Phys. Rev. B 98, 035302 (2018).

[52] S. Rudin, T. L. Reinecke, and B. Segall, Temperature-dependent exciton linewidths in semiconductors, Phys. Rev. B 42, 11218 (1990).

[53] S. Brem, J. Zipfel, M. Selig, A. Raja, L. Waldecker, J. D. Ziegler, T. Taniguchi, K. Watanabe, A. Chernikov, and E. Malic, Intrinsic lifetime of higher excitonic states in tungsten diselenide monolayers, Nanoscale 11, 12381 (2019).

[54] V. Chellappan, A. L. C. Pang, S. Sarkar, Z. E. Ooi, and K. E. J. Goh, Effect of phonons on valley depolarization in monolayer $\mathrm{WSe}_{2}$, Electron. Mater. Lett. 14, 766 (2018).

[55] G. Wang, X. Marie, L. Bouet, M. Vidal, A. Balocchi, T. Amand, D. Lagarde, and B. Urbaszek, Exciton dynamics in $\mathrm{WSe}_{2}$ bilayers, Appl. Phys. Lett. 105, 182105 (2014). 